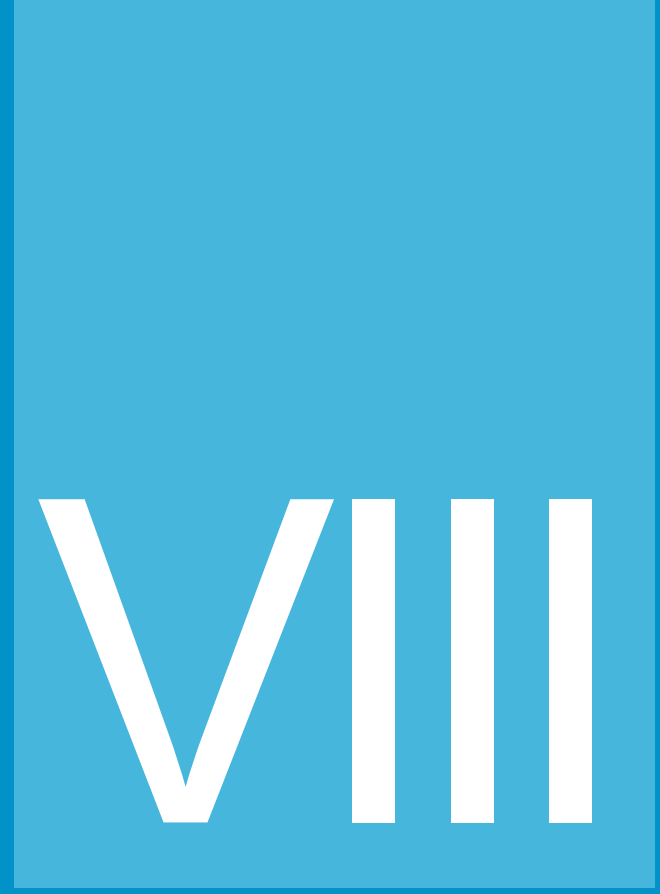

\title{
LA ENFERMEDAD DEL SIGLO XXI Y LA CALIDAD DE VIDA EN ADULTOS MAYORES DE TARAPOTO
}

The XXI century disease and quality of life in older adults in Tarapoto

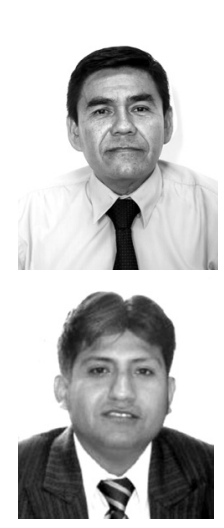

Andrés Bartra

Bachiller en Psicología por la Universidad Peruana Unión. Licenciado en Educación por la Universidad Nacional Mayor de San Marcos. Investigador y conferenciante. Actualmente se desempeña como docente en centros educativos pertenecientes al Ministerio de Educación del Perú.

\section{Renzo Carranza}

Licenciado en Psicología por la Universidad Peruana Unión. Docente Auxiliar de la Facultad de Ciencias de la Salud por la misma universidad. Investigador y docente visitante. Actualmente se desempeña como director de investigación de la Escuela Académica Profesional de Psicología en la sede de Tarapoto de la Universidad Peruana Unión. Conferenciante a nivel nacional e internacional. 


\section{Resumen}

A diario resulta interesante poder observar que el envejecimiento de la población a nivel mundial va en aumento, y esto ha despertado el interés por conocer más de cerca las emociones como también la calidad de vida de las personas mayores. El objetivo de este estudio fue determinar en qué medida están relacionadas la depresión y la calidad de vida en los adultos mayores del Puesto de Salud Huayco - Tarapoto. Se utilizó un diseño no experimental de tipo descriptivo correlacional. Instrumentos: Inventario de depresión de Beck (BDI-II) conformada por 21 items y el Formato de Evaluación Integral del Adulto Mayor del Ministerio de Salud (sección de Calidad de Vida), conformada por 33 ítems. Las propiedades psicométricas de los instrumentos indicaron que son válidos y confiables. Los resultados evidencian que existe una relación negativa y altamente significativa entre la depresión y la calidad de vida $(r=-0.432, p<0.01)$. Esto quiere decir que cuanto mayor sea la depresión, la calidad de vida se verá afectada.

Palabras clave: Depresión, calidad de vida, adultos mayores.

\section{Abstract}

Every day is interesting to observe that the aging of the global population is increasing and this has led to interest in learning more about the emotions as well as the quality of life of older people. The aim of this study was to determine to what extent are related depression and quality of life in older adults Huayco Health Center - Tarapoto. We used a non-experimental, descriptive correlational. Instruments: Beck Depression Inventory (BDI-II) consists of 21 items and Comprehensive Evaluation Form Senior Ministry of Health (Quality of Life section), consisting of 33 items. The psychometric properties of the instruments indicated that are valid and reliable. The results show that there is a highly significant negative relationship between depression and quality of life $(r=-0.432, p<.01)$. This means that, how much more can be the depression then quality of life will be affected.

Keywords: Depression, quality of life, seniors. 


\section{Introducción}

En años pasados la depresión era considerada como un signo, un síntoma o un síndrome; sin embargo, en la actualidad es una enfermedad como cualquier otra que ataca sin hacer distinciones de edad, sexo, estado civil, nivel socioeconómico, etc. Así también, hablar de depresión es relacionar este término con el imán emocional que atrae y une el desaliento, la soledad, incomprensión, amargura y/o tristeza en un momento determinado, en la existencia de una persona.

Por otro lado, a través de los años, el interés por la calidad ha existido desde hace mucho tiempo, sin embargo, calidad de vida como tal es un término reciente y está llegando a ser muy importante en los estudios actuales. A diario resulta interesante poder observar que el envejecimiento de la población a nivel mundial va en aumento y esto ha despertado el interés por conocer más de cerca las emociones como también la calidad de vida de las personas mayores.

De otro lado, investigaciones realizadas por la Organización Mundial de la Salud (OMS, 2011), el nivel de depresión que afecta al mundo es alarmante, por tanto, la calidad de vida de la población se ve afectada en las mismas dimensiones. Se ha determinado que un promedio de 350 millones de personas la padecen actualmente. Es la principal causa mundial de discapacidad y afecta más a la mujer que al hombre. Hay tratamientos eficaces para la depresión, pero un promedio de $90 \%$ de personas afectadas no utilizan dichos servicios.

Está previsto que en la segunda década del presente milenio de acuerdo a la Organización Mundial de la Salud (2001, citado por Pereyra y Mussi, 2005) se prevé que para el año 2020 la depresión será la segunda enfermedad más importante de incapacitación y muerte, después de las enfermedades cardiovasculares; asimismo, estudios realizados en diversos países de Latinoamérica revelan que Brasil es el país que presenta el más alto índice de depresión en su población, seguido de Chile, Colombia, México y Argentina, los cuales han implementado políticas de corto, mediano y largo plazo para la atención de la salud mental. Ello implica que se está dedicando recursos importantes para atender esta problemática, teniendo como uno de sus factores contribuyentes al desarrollo de cada nación.

En nuestro país, el Instituto Especializado de Salud Mental "Honorio Delgado-Hideyo Noguchi realizó un estudio epidemiológico el año 2008 en Lima Metropolitana, encontrando prevalencias de vida para episodio depresivo de 18,2 \%. En la sierra, las ciudades de Huaraz, Cajamarca y Ayacucho se encontró una prevalencia de vida de episodio depresivo de 16,2 \%, mientras 
que en Iquitos, Pucallpa y Tarapoto, se encontró una prevalencia de vida de 21,4 \% (Alarcón, 2010), elevados indicadores de depresión. Es preciso también mencionar que en la jurisdicción regional de San Martín, los indicadores señalan que más de la mitad de su población experimenta signos y síntomas depresivos, siendo las mujeres las que más la padecen, hecho que incide en un inadecuado desarrollo personal en sus diversas connotaciones (Dirección Regional de Salud, 2011); asimismo, el porcentaje de adolescentes que ha llegado a desarrollar cuadros depresivos es notable, registrándose casos de suicidio, principalmente en escolares.

Cabe mencionar que las instituciones asistenciales de salud, a nivel de sus microrredes cuentan con un sistema de atención orientado a mejorar la calidad de vida de la población, cuyo estado actual es preocupante y se deriva como consecuencia del nivel socioeconómico y cultural en que se desenvuelven. Asimismo, tienen una gran responsabilidad social frente a los diferentes grupos etarios vulnerables, y deberán mejorar el servicio que prestan, porque la realidad así lo amerita.

Por otro lado, el nivel socioeconómico de la población adulta mayor del barrio Huayco-Tarapoto, en la cual estuvo centrada la investigación, se ubica en un nivel "bajo", siendo las áreas urbano-marginales las más susceptibles de Tarapoto.

A continuación se describen y definen conceptualmente las variables que configuran nuestro problema de investigación.

\section{La depresión}

En los últimos años la depresión ha sido catalogada como uno de los problemas que más sufrimiento causa en las personas y que en distintos grados afecta a un porcentaje muy alto de la población sin diferencia de edad, género, nivel socioeconómico, convirtiéndola en uno de los principales motivos de consulta clínica. La depresión puede ser desencadenada por cualquier cambio de vida estresante, incluso si es un cambio drástico en la vida normal y habitual; de otro lado, es importante diferenciar la depresión de la tristeza. La tristeza es un estado de ánimo pasajero, provocado por una situación concreta, como por ejemplo, la muerte de un ser querido. Cuando este estado de ánimo perdura en el tiempo, podemos estar frente a una depresión. Según National Institute of Mental Healt (2001) refiere que los siguientes síntomas pueden considerarse como indicadores de la depresión: Un estado de ánimo triste o ansioso persistente al igual que sensaciones de desesperanza, el pesimismo, la 
culpabilidad, la impotencia, la pérdida de interés en actividades que resultaban ser placenteras, el cansancio -fatiga-, la energía se encuentra disminuida, los problemas de concentración y memoria, la dificultad para tomar decisiones, la autocrítica, el despertarse en la madrugada o dormir demasiado, los cambios en el apetito, aquellos pensamientos de muerte o intentos de suicidio, la inquietud, la irritabilidad como también los dolores de cabeza y alteraciones digestivas que no responden a tratamiento médico; son síntomas que se pueden considerar como indicadores de la depresión.

\section{Definición de depresión}

Respecto a la depresión, Beck (1976) menciona que la depresión se debe a un desorden del pensamiento, en el que el depresivo distorsiona la realidad, viéndose de forma negativa en la triada cognitiva: él mismo, el mundo y el futuro, además supone la activación de signos y síntomas como consecuencia de la activación de patrones cognitivos negativos.

Por otro lado, Robert y Lamontegne (1977) consideran que la depresión es un estado de tristeza, acompañado de una marcada disminución del sentimiento de valor personal de una dolorosa consecuencia de disminución de actividad mental psicomotriz y orgánica, sin embargo, Fremman y Oster (1984; citados por Salmuri y Skoknic, 2005) postulan que la depresión es un trastorno conocido desde hace tiempo. Se dice que es el problema psicológico de nuestros días, considerado como el resfriado de los trastornos emocionales; asimismo, para Cottraux (1988; citado por Vázquez y Sanz, 1999) la depresión es una síndrome caracterizado por un estado de ánimo que se traduce en la expresión verbal y no verbal de sentimientos tristes, acompañados de ansiedad, irritabilidad, dichas características pueden alterar múltiples funciones, el sueño y el apetito están aumentados o disminuidos, la energía y el impulso sexual se encuentran disminuidos, la motivación se traduce en un déficit del interés y de la capacidad para experimentar placer, el deseo de evadirse mediante el suicido es frecuente, la evitación de los contactos sociales y la dependencia respecto a los demás lleva recientemente a un rechazo por el medio. Los comportamientos activos y productivos se encuentran disminuidos y los pensamientos negativos se encuentran incrementados.

\section{Calidad de vida}

La Organización Mundial de la Salud (2010) define a calidad de vida como la satisfacción que experimentan los individuos como resultado de su 
participación en las actividades que realizan en el medio familiar, en el centro de trabajo y en el ámbito comunal y nacional, en relación con los cuales se ejercitan sus capacidades humanas y desarrollan su personalidad. Asimismo, calidad de vida es un expresión lingüística cuyo significado es eminentemente subjetivo; está asociado con la personalidad de la persona, con su bienestar y la satisfacción por la vida que lleva, y cuya evidencia está intrínsicamente relacionada a su propia experiencia, a su salud y a su grado de interacción social y ambiental y, en general, a múltiples factores.

Por otro lado, Velandia (1994) refiere que la calidad de vida del adulto mayor es el resultado de la interacción entre las diversas características de la vida humana tales como la alimentación, educación, vivienda, entre otras; cada una de ellas es significativa en la existencia humana, pues permite un óptimo bienestar, teniendo en cuenta el proceso evolutivo del envejecimiento, las adaptaciones del ser humano a su entorno, las cuales se dan en forma personal y diferentes; que influyen estas en su salud física, emocional, mental y espiritual. De otro lado, O'Shea (2003; citado por Lapuente, 2011) afirma que la calidad de vida del adulto mayor es una vida satisfactoria, bienestar subjetivo y psicológico, desarrollo personal y diversas representaciones de lo que constituye una buena vida. Asimismo, Krzeimen (2001; citado por Vera, 2007) señala que la calidad de vida del adulto mayor se da en la medida que este logre el reconocimiento a partir de relaciones sociales significativas; entonces, a partir de estas experiencias su ser se sentirá con ganas de continuar el proceso vital; de lo contrario, se vivirá como una fase de declinación funcional y aislamiento social.

Por lo tanto, consideramos que la depresión y la calidad de vida en los adultos mayores del Puesto de Salud Huayco - Tarapoto están interrelacionadas.

\section{Método}

\section{Diseño y tipo de investigación}

La investigación corresponde a un diseño no experimental o ex-postfacto, de tipo correlacional porque se buscó encontrar la relación entre la depresión y calidad de vida en los adultos mayores del Puesto de Salud HuaycoTarapoto (Hernández, Fernández y Baptista, 2010).

\section{Muestra}

Estuvo conformada por 82 adultos mayores del sexo masculino y femenino, atendidos en el Puesto de Salud Huayco - Tarapoto. 


\section{Variables}

Depresión y Calidad de Vida.

\section{Instrumentos}

a. Inventario de depresión de Beck (BDI-II) construida por Beck, Steer y Brown (2006) y conformada por 21 ítems.

b. Formato de Evaluación Integral del Adulto Mayor del Ministerio de Salud (sección de Calidad de Vida), conformada por 33 ítems.

Para el análisis de datos se utilizaron Técnicas Estadísticas Descriptivas e inferenciales Correlación de Pearson, a un nivel de significancia $p<0.05$. Para procesar los datos se utilizó el Software Estadístico SPSS.

\section{Resultados}

Propiedades psicométricas del Inventario de Depresión de Beck (BDI-II)

\section{Confiabilidad}

En cuanto a determinar la confiabilidad en su modalidad de consistencia interna se estimó el coeficiente Alfa de Cronbach, obteniéndose como resultado para la escala total un valor de 0.790 para el Inventario de Depresión de Beck (Tabla 1), lo que indica que el instrumento permite obtener puntajes confiables.

Tabla 1.- Estimaciones de confiabilidad mediante el coeficiente Alfa de Cronbach para el Inventario de Depresión de Beck (BDI-II).

\begin{tabular}{cc}
\hline $\mathrm{N}^{\circ}$ de ítems & Alfa de Cronbach \\
\hline 21 & 0.790 \\
\hline
\end{tabular}

Como se puede observar en la Tabla 2, el coeficiente Alfa de Cronbach, arroja un valor de 0.841 para la Encuesta de Uso de Chat, lo que indica que el instrumento es confiable. 


\section{Validez}

Como logramos apreciar en la Tabla 2, la Prueba KMO y Prueba de Barlett, en conclusión, valores bajos del índice KMO desaconsejan la utilización de Análisis Factorial. En la Tabla 2 se observa que el índice KMO es de 0.778, siendo un valor meritorio para obtener el análisis factorial, lo que indica que el instrumento presenta validez.

Tabla 2.- Prueba KMO y prueba de Barlett

\begin{tabular}{|c|c|c|}
\hline \multicolumn{3}{|c|}{ KMO y prueba de Bartlett } \\
\hline $\begin{array}{l}\text { Medida de adecuación } \\
\text { Meyer-Olkin. }\end{array}$ & muestral de Kaiser- & ,778 \\
\hline \multirow[t]{3}{*}{$\begin{array}{l}\text { Prueba de esfericidad } \\
\text { de Bartlett }\end{array}$} & $\begin{array}{l}\text { Chi-cuadrado aproxi- } \\
\text { mado }\end{array}$ & $1,401,681$ \\
\hline & gl & 528 \\
\hline & Sig. & 000, \\
\hline
\end{tabular}

\section{Propiedades psicométricas del Formato de Evaluación Integral del Adulto Mayor del Ministerio de Salud}

Se aprecia en la Tabla 3 que el coeficiente Alfa de Cronbach muestra como resultado 0.710 para el Formato de Evaluación Integral del Adulto Mayor del Ministerio de Salud, lo indica que el instrumento es confiable.

Tabla 3.- Estimaciones de confiabilidad mediante el coeficiente Alfa de Cronbach para el Formato de Evaluación Integral del Adulto Mayor del Ministerio de Salud

\begin{tabular}{cc}
\hline $\mathrm{N}^{\circ}$ de ítems & Alfa de Cronbach \\
\hline 33 & 0.710 \\
\hline
\end{tabular}

Se puede apreciar en la Tabla 4, referente a la Prueba KMO y Prueba de Barlett, que el índice KMO es de 0.752 siendo un valor meritorio para obtener el análisis factorial, lo que indica que el instrumento presenta validez. 


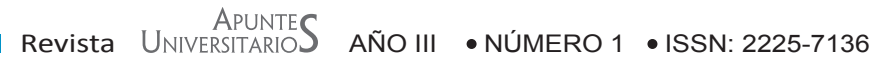

Tabla 4.- Prueba KMO y prueba de Barlett

\begin{tabular}{|c|c|c|}
\hline \multicolumn{3}{|c|}{ KMO y prueba de Bartlett } \\
\hline $\begin{array}{l}\text { Medida de adecuación } \\
\text { Olkin }\end{array}$ & nuestral de Kaiser-Meyer- & 752 \\
\hline \multirow{3}{*}{$\begin{array}{l}\text { Prueba de esfericidad } \\
\text { de Bartlett }\end{array}$} & Chi-cuadrado aproximado & 462,179 \\
\hline & $\mathrm{gl}$ & 36 \\
\hline & Sig. & ,000 \\
\hline
\end{tabular}

\section{Análisis descriptivo de las variables de estudio}

Se percibe en la Tabla 5 que un $68.3 \%$ de la población de adultos mayores del Centro de Salud Huayco pertenece al género femenino; de otro lado, se aprecia que un $50 \%$ de adultos mayores se ubica entre las edades de 66 y 75 años de edad y quienes presentan mayores niveles de depresión.

Tabla 5.- Niveles de depresión por características demográficas (porcentaje entre paréntesis)

\begin{tabular}{|c|c|c|c|c|c|c|c|c|c|c|}
\hline \multirow{2}{*}{ Características } & \multicolumn{10}{|c|}{ Nivel de depresión } \\
\hline & \multicolumn{2}{|c|}{ Mínima } & \multicolumn{2}{|c|}{ Leve } & \multicolumn{2}{|c|}{ Moderada } & \multicolumn{2}{|c|}{ Severa } & \multicolumn{2}{|c|}{ Total } \\
\hline Masculino & 5 & $(6.1)$ & 17 & $(20.7)$ & 2 & $(2.4)$ & 2 & (2.4) & 26 & (31.7) \\
\hline Femenino & 5 & $(6.1)$ & 32 & (39.0) & 17 & $(20.7)$ & 2 & (2.4) & 56 & (68.3) \\
\hline \multicolumn{11}{|l|}{ Edad } \\
\hline $60-65$ & 5 & (6.1) & 3 & (3.7) & 4 & $(4.9)$ & 1 & (1.2) & 13 & (15.9) \\
\hline $66-70$ & 3 & (3.7) & 13 & $(15.9$ & 4 & (4.9) & 0 & $(0.0)$ & 20 & (24.4) \\
\hline $71-75$ & 0 & $(0.0)$ & 18 & $(22.0)$ & 3 & $(3.7)$ & 0 & $(0.0)$ & 21 & (25.6) \\
\hline $76-80$ & 1 & $(1.2)$ & 9 & $(11.0)$ & 6 & $(7.3)$ & 0 & $(0.0)$ & 16 & (19.5) \\
\hline $81-85$ & 1 & $(1.2)$ & 3 & (3.7) & 1 & $(1.2)$ & 2 & (2.4) & 7 & $(8.5)$ \\
\hline $86-90$ & 0 & (0) & 3 & (3.7) & 1 & $(1.2)$ & 1 & (1.2) & 5 & (6.1) \\
\hline \multicolumn{11}{|l|}{ Religión } \\
\hline Adventista & 0 & $(0.0)$ & 5 & (6.1) & 2 & (2.4) & 0 & $(0.0)$ & 7 & (8.5) \\
\hline Católico & 6 & (7.3) & 39 & $(47.6)$ & 12 & $(14.6)$ & 3 & (3.7) & 60 & (73.2) \\
\hline Evangélico & 3 & (3.7) & 4 & (49.9) & 2 & $(2.4)$ & 1 & $(1.2)$ & 10 & (12.2) \\
\hline Testigo de Jehová & 1 & $(1.2)$ & 0 & $(0.0)$ & 0 & $(0.0)$ & 0 & $(0.0)$ & 1 & $(1.2)$ \\
\hline Otros & 0 & $(0.0)$ & 1 & $(1.2)$ & 3 & $(3.7)$ & 0 & $(0.0)$ & 4 & (4.9) \\
\hline Total & 10 & $(12.2)$ & 49 & $(59.8)$ & 19 & $(23.2)$ & 4 & (4.9) & 82 & (100) \\
\hline
\end{tabular}

Respecto a la calidad de vida, en la Tabla 06 se observa que un $68.3 \%$ de la población de adultos mayores del Centro de Salud Huayco pertenece al 
género femenino quienes presentan una regular calidad de vida; de otro lado, se aprecia que un $50 \%$ de adultos mayores se ubica entre las edades de 66 y 75 años de edad y quienes presentan una calidad de vida regular.

Tabla 6.- Niveles de calidad de vida por características demográficas (porcentaje entre paréntesis)

\begin{tabular}{|c|c|c|c|c|c|c|c|c|}
\hline \multirow{2}{*}{$\begin{array}{l}\text { Características } \\
\text { Sexo }\end{array}$} & \multicolumn{2}{|c|}{ Mala } & \multicolumn{2}{|c|}{ Regular } & \multicolumn{2}{|c|}{ Buena } & \multicolumn{2}{|c|}{ Total } \\
\hline & & & & & & & & \\
\hline Masculino & 2 & (2.4) & 21 & $(25.6)$ & 3 & (3.7) & 26 & (31.7) \\
\hline Femenino & 0 & $(0.0)$ & 50 & $(61.0)$ & 6 & $(7.3$ & 56 & (68.3) \\
\hline \multicolumn{9}{|l|}{ Edad } \\
\hline $60-65$ & 0 & $(0.0)$ & 12 & $(14.6)$ & 1 & $(1.2)$ & 1 & (15.8) \\
\hline $66-70$ & 1 & $(1.2)$ & 17 & $(20.7)$ & 2 & (2.4) & 2 & (24.4) \\
\hline $71-75$ & 0 & $(0.0)$ & 20 & $(24.4)$ & 1 & $(1.2)$ & 1 & (25.6) \\
\hline $76-80$ & 1 & $(1.2)$ & 14 & (17.1) & 1 & $(1.2)$ & 1 & (19.5) \\
\hline $81-85$ & 0 & $(0.0)$ & 5 & $(6.1)$ & 2 & (2.4) & 2 & (8.5) \\
\hline $86-90$ & 0 & $(0.0)$ & 3 & $(4.2)$ & 2 & $(22.2)$ & 2 & (6.1) \\
\hline \multicolumn{9}{|l|}{ Religión } \\
\hline Adventista & 0 & $(0.0)$ & 7 & $(8.5)$ & 0 & $(0.0)$ & 7 & (8.5) \\
\hline Católico & 2 & (2.4) & 51 & $(62.2)$ & 7 & (8.5) & 60 & (73.2) \\
\hline Evangélico & 0 & $(0.0)$ & 8 & $(9.8)$ & 2 & (2.4) & 10 & (12.2) \\
\hline Testigo de Jehová & 0 & $(0.0)$ & 1 & $(1.2)$ & 0 & $(0.0)$ & 1 & $(1.2)$ \\
\hline Otros & 0 & $(0.0)$ & 4 & $(5.6)$ & 0 & $(0.0)$ & 4 & (4.9) \\
\hline Total & 2 & (2.4) & 71 & $(86.6)$ & 9 & (11.0) & 82 & (100) \\
\hline
\end{tabular}

\section{Relación entre depresión y calidad de vida}

Como se puede apreciar en la Tabla 7, el coeficiente de correlación ( $r$ ) nos indica que existe una relación negativa y altamente significativa entre la depresión y la calidad de vida $(r=-0.432, p<0.00)$. Esto quiere decir que cuanto mayor sea el nivel de depresión, la calidad de vida será mala.

Tabla 7.- Análisis de correlación entre depresión y calidad de vida

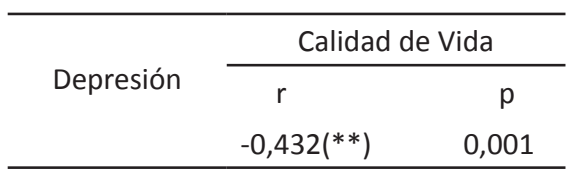

** La correlación es significativa al nivel 0,01 (bilateral) 


\section{Resultados sobre la relación entre la depresión y las dimensiones de calidad de vida}

En la Tabla 8 se muestran los coeficientes de correlación ( $r$ ) para la variable depresión y las dimensiones de la variable calidad de vida obtenidos para la muestra total. En dicha Tabla se observa que las correlaciones entre la depresión y calidad de vida son negativas y altamente significativas, observándose, además, que cuanto mayor sea la depresión, el estado mental será menor $\left(r=-.320^{* *} ; p=.000\right)$, asimismo, se aprecia que a mayor nivel de depresión el aspecto sociofamiliar será constreñido ( $\left.r=-.336^{* *} ; p=.002\right)$, así también cuanto mayor sea la depresión, menor será la valoración espiritual ( $r=-.315^{*}$; $\mathrm{p}=.005)$, finalmente cuanto mayor sea la depresión, menor será la salud física $(r=-.452 * * ; p=.000)$.

Tabla 8.- Coeficientes de correlación entre la depresión y las dimensiones de calidad de vida

\begin{tabular}{lcc}
\hline Dimensiones calidad de vida & \multicolumn{2}{c}{ Depresión } \\
\hline & $\mathrm{r}$ & $\mathrm{p}$ \\
\cline { 2 - 3 } Estado mental & $-0.320^{* *}$ & .000 \\
Socio-familiar & $-0.336^{* *}$ & .002 \\
Espiritual & $-0.315^{*}$ & .005 \\
Salud Física & $-0.452^{* *}$ & .000 \\
\hline ** La correlación es significante a nivel 0.01 &
\end{tabular}

\section{Discusión}

A tenor de los resultados obtenidos, cabe señalar que la población anciana ha crecido en estos últimos años y se considera un crecimiento mayor a nivel mundial, de acuerdo a las proyecciones, en el Perú, para el año 2020, el $11 \%$ de la población total estará compuesta por ancianos (Chinga, 2009).

La importancia de este trabajo radica en la evidencia empírica que aporta al estudio de la calidad de vida, demostrando una correlación negativa altamente significativa $(p<0.001)$ entre depresión y calidad de vida; indicando que cuanto mayor sea la depresión, la calidad de vida se verá afectada.

Las investigaciones realizadas por la Organización Mundial de la Salud (2011) indica que la depresión que afecta al mundo es alarmante, por tanto, la calidad de vida de la población se ve afectada en las mismas dimensiones, 
en tal sentido otra de la contribuciones de mucha importancia del presente estudio es que los adultos mayores del barrio Huayco presentan niveles de depresión, resultados similares se evidencian en el estudio realizado por Gonzales (2001) quien encontró que existe depresión en los ancianos de la provincia de Vizcaya (50\%).

Otro hallazgo muy importante, en este estudio, es la confirmación de que la calidad de vida de los adultos mayores del barrio Huayco es regular; los resultados de nuestra investigación se asemeja un tanto a los resultados encontrados por Pérez, et al. (2003) quienes encontraron que la calidad de vida de los pacientes ancianos en hemodiálisis es regular y mala.

Renzo Carranza

Universidad Peruana Unión email: renzo.carranza@upeu.edu.pe

Andrés Bartra

Universidad Peruana Unión email: karl.fam@hotmail.com

Recibido: 26 de febrero de 2013 Aceptado: 16 de abril de 2013 


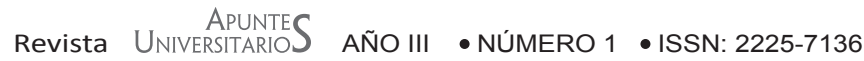

\section{Referencias}

Alarcón, C. (2010). Depresión en el Adulto Mayor. Revista Peruana de Epidemiología, 14(2), 14-20.

Beck, A., Steer, R., GK, Brown. (2006). Inventario de Depresión de Beck. Argentina: Paidos.

Beck, A. T., Rush, J., Shaw, B., y Emery, G. (1979). Terapia cognitiva de la depresión. Bilbao: DDB.

Chinga, M. (2009). Síndromes geriátricos del paciente adulto mayor hospitalizado en el Hospital Nacional Arzobispo Loayza: noviembre-diciembre 2008. Tesis inédita de Licenciatura. Universidad Nacional Mayor de San Marcos, Lima.

Gareces, S. (2011). Aproximación cualitativa a las percepciones, creencias, actitudes y necesidades de la población jubilada en un ámbito rural. Recuperado de http://zaguan.unizar.es/ TAZ/ENFHUES/2012/7142/TAZ-TFG-2012-014.pdf

Gonzales, M. (2001). Depresión en ancianos: Un problema de todos. Revista Cubana de medicina general. 17(4), 316-320.

Hernández, R., Fernández, C., y Baptista, P. (2010). Metodología de la investigación. México: Mc Graw- Hill.

Krzemien D. (2001). Calidad de vida y participación social en la vejez femenina. Grupo de investigación psicológica del desarrollo, 1,1, 3-10.

Pérez, V., Borrego, J., Viedma, G., Gil, J., Ortega, S., Forondam J., Sanchez, M., Pérez del Barrio, P., García, M., Borrego, J. y Liebana, A. (2003). Calidad de vida relacionada con la salud en pacientes ancianos en hemodiálisis. Nefrología: publicación oficial de la Sociedad Española de Nefrología, 23(6), 528-537

Pereyra, M. y Mussi, C. (2005). Sea Feliz, ¿cómo vencer la depresión y controlar la ansiedad? Montemorelos, Nuevo León: Ed. Montemorelos México.

Robert, S. y Lamontagne, Y. (1977). Depresión. Principios y aplicaciones de las terapias de la conducta. Madrid: Debate.

Salmuri, F. y Skoknic, V. (2005). Efectos conductuales de la educación emocional en alumnos de educación básica. Revista de Psicología Universidad de Chile, 14(001), 9-28.

Vázquez, C. y Sanz, J. (1999). Fiabilidad y validez de la versión española del Inventario para la Depresión de Beck de 1978 en pacientes con trastornos psicológicos. Revista especializada en la psicología clínica y de la salud: trastornos psicológicos, saludenfermedad, prevención y atención a los diferentes niveles asistenciales (primaria, secundaria y terciaria), 1(10), 59-81.

Vera, M. (2007). Significado de la calidad de vida del adulto mayor para sí mismo y para su familia. Revista Anales de la Facultad de Medicina, 68(3), 284-290. 


\section{Andrés Bartra - Renzo Carranza}

\section{Enlaces}

National Institute of Mental Health. (2002). Depresión. Recuperado de http://www.nimh.nih. gov/publicat/spDep3561.cfm

Organización Mundial de la Salud. (2011). La depresión es una enfermedad frecuente y las personas que la padecen necesitan apoyo y tratamiento. Recuperado de http://www.who.int/ mediacentre/news/notes/2012/mental_health_day_20121009/es/index.html

Organización Mundial de la Salud. (2010). Calidad de Vida. Recuperado de http://www.who.int/ calidaddevida/2010/12/oms-calidad-de-vida-2005.html 